\title{
On Using Curvature to Demonstrate Stability
}

\author{
C. Connell McCluskey \\ Department of Mathematics, Wilfrid Laurier University, 75 University Ave West, \\ Waterloo, ON, Canada N2L 3C5 \\ Correspondence should be addressed to C. Connell McCluskey, ccmcc8@gmail.com
}

Received 11 September 2007; Revised 28 February 2008; Accepted 2 April 2008

Recommended by Bhabani Dandapat

A new approach for demonstrating the global stability of ordinary differential equations is given. It is shown that if the curvature of solutions is bounded on some set, then any nonconstant orbits that remain in the set, must contain points that lie some minimum distance apart from each other. This is used to establish a negative-criterion for periodic orbits. This is extended to give a method of proving an equilibrium to be globally stable. The approach can also be used to rule out the sudden appearance of large-amplitude periodic orbits.

Copyright ( 12008 C. Connell McCluskey. This is an open access article distributed under the Creative Commons Attribution License, which permits unrestricted use, distribution, and reproduction in any medium, provided the original work is properly cited.

\section{Introduction}

A key issue in the analysis of a system of ordinary differential equations is to determine the long-term dynamics. Generally, it is desirable, if possible, to know whether a system settles down to an equilibrium, approaches a limit cycle, or exhibits some other long-term behavior. In this paper, we give a method that uses the curvature of solutions to help show that a system approaches an equilibrium.

A nice feature of considering the curvature is that the calculation only involves local information. Furthermore, it is not necessary to calculate solutions to the differential equations since the curvature calculation uses the derivative of a solution, and this is given by the vector field that defines the flow.

On any set for which the curvature is bounded, there is a minimum length of a closed orbit (not including equilibria). Also, in such a set, any closed orbit must contain points that are a certain distance away from each other. The smaller the curvature, the farther apart certain points on the orbit must be from each other. This idea is used to formulate a negative criterion for periodic orbits. The method is then extended to give a criterion that shows that all forward trajectories leave the set. 
Unfortunately, if the eigenvalues of the Jacobian at an equilibrium are not all equal, then the curvature near the equilibrium is unbounded. This makes it necessary to deal with the behavior near an equilibrium separately. For instance, one may use a Lyapunov function near an equilibrium to show that a certain neighborhood is contained in the equilibrium's basin of attraction. Then, analysis of the curvature can be used to show that all other solutions enter this set (by showing that they leave the complement), making the stability global.

This approach can also be useful in ruling out nonlocal bifurcations as parameters are varied. It is often possible to show that an equilibrium is locally asymptotically stable for all parameter values, and that it is globally stable for a particular set of parameter values. As parameters are varied, the continued local stability precludes the possibility of local bifurcations such as Hopf. Nonlocal bifurcations, however, may still occur. The method presented here can be used to preclude the existence (and sudden appearance) of largeamplitude periodic orbits and, therefore, help rule out nonlocal bifurcations.

We now give a brief outline of the paper. In Section 2, the curvature of parametric curves is discussed. This is applied to periodic solutions of differential equations in Section 3. In Section 4, results are given for omega limit points. Section 5 stability gives stability theorems. A discussion of how to apply the results is included in Section 6.

\section{Curvature Calculations}

Consider a curve $\Gamma$ given by the function $r: \mathbb{R} \rightarrow \mathbb{R}^{n}$, where $r$ is $C^{2}$ and $r^{\prime}$ is nonzero for all $t \in \mathbb{R}$. In this paper, we use the convention that $\left({ }^{\prime}\right)$ represents differentiation with respect to time.

Let $s$ be the arclength along $\Gamma$ from some reference point, that is, $s(t)=\int_{t_{0}}^{t}\left\|r^{\prime}(\tau)\right\| d \tau$. We use the convention that when the arguement of a function is changed to $s$, the function is considered to be reparameterized in terms of arc length. Note that $d s / d t=\left\|r^{\prime}(t)\right\|$, and so $d / d s=(d / d t)(d t / d s)=\left(1 /\left\|r^{\prime}(t)\right\|\right)(d / d t)$.

For each $t$, the unit tangent at the point $r(t)$ is

$$
T(t)=\frac{r^{\prime}(t)}{\left\|r^{\prime}(t)\right\|}
$$

and so

$$
\begin{aligned}
T^{\prime}(t) & =\frac{\left\|r^{\prime}\right\| r^{\prime \prime}-\left(\left(1 /\left\|r^{\prime}\right\|\right) r^{\prime} \cdot r^{\prime \prime}\right) r^{\prime}}{\left\|r^{\prime}\right\|^{2}} \\
& =\frac{\left\|r^{\prime}\right\|^{2} r^{\prime \prime}-\left(r^{\prime} \cdot r^{\prime \prime}\right) r^{\prime}}{\left\|r^{\prime}\right\|^{3}} .
\end{aligned}
$$

The curvature [1] of $\Gamma$ is defined to be $K=\|d T / d s\|$ and is given by

$$
K=\frac{\left\|T^{\prime}(t)\right\|}{\left\|r^{\prime}(t)\right\|} .
$$


Using (2.2) gives

$$
K=\frac{\|\| r^{\prime}\left\|^{2} r^{\prime \prime}-\left(r^{\prime} \cdot r^{\prime \prime}\right) r^{\prime}\right\|}{\left\|r^{\prime}(t)\right\|^{4}}
$$

Let $T_{0}$ be a unit vector in $\mathbb{R}^{n}$. Let $\theta(t)$ be the angle between $T(t)$ and $T_{0}$ :

\section{Proposition 2.1.}

$$
\left|\frac{d \theta}{d s}\right| \leq K
$$

Proof. Noting that $\cos (\theta)=T_{0} \cdot T$, we have $-\sin (\theta)(d \theta / d s)=T_{0} \cdot d T / d s$. Letting $\beta$ be the angle between $d T / d s$ and $T_{0}$, then, $T_{0} \cdot d T / d s=\|d T / d s\| \cos (\beta)$. Thus,

$$
\begin{aligned}
\frac{d \theta}{d s} & =-\frac{1}{\sin (\theta)} T_{0} \cdot \frac{d T}{d s} \\
& =-\frac{1}{\sin (\theta)}\left\|\frac{d T}{d s}\right\| \cos (\beta) \\
& =-\frac{\cos (\beta)}{\sin (\theta)} K .
\end{aligned}
$$

We now show that $|\cos (\beta) / \sin (\theta)|$ is at most one. Since $T(s)$ is a unit vector for all $s$, it follows that $d T / d s$ is orthogonal to $T$. Since $T_{0}$ is a unit vector separated from $T$ by angle $\theta$, the component of $T_{0}$ normal to $T$ has magnitude $\sin (\theta)$. Thus, the component of $T_{0}$ in the direction of $d T / d s$ has magnitude less than or equal to $\sin (\theta)$. On the other hand, this magnitude is given by $|\cos (\beta)|$, showing that $|\cos (\beta) / \sin (\theta)| \leq|\sin (\theta) / \sin (\theta)|=1$. Combining with (2.6) yields the proposition.

Now, suppose $\Gamma$ is a simple closed curve given by the $C^{2} \omega$-periodic function $r: \mathbb{R} \rightarrow \mathbb{R}^{n}$, where $r^{\prime}$ is nonvanishing. Let $x_{0} \in \Gamma$. Choose $t_{0} \in \mathbb{R}$ such that $r\left(t_{0}\right)=x_{0}$, and let $T_{0}=T\left(t_{0}\right)$, that is, $T_{0}$ is the unit tangent to $\Gamma$ at $x_{0}$.

Since $r$ is $\omega$-periodic, it follows that $0=r(t+\omega)-r(t)=\int_{t}^{t+\omega} r^{\prime}(\tau) d \tau$ for all $t$. Hence, $0=\int_{t}^{t+\omega}\left(r^{\prime}(\tau) \cdot T_{0}\right) d \tau$. Combining this with the fact that $\left(r^{\prime}(t) \cdot T_{0}\right)$ is $\omega$-periodic, continuous and positive at $t=t_{0}$, we see that $\left(r^{\prime}(t) \cdot T_{0}\right)$ oscillates in sign and, therefore, must have at least two zeros in every interval of length $\omega$. Let $\left(t^{-}, t^{+}\right)$be the maximal interval containing $t_{0}$ such that $\left(r^{\prime}(t) \cdot T_{0}\right)$ is positive. Note that $\theta\left(t^{-}\right)=\theta\left(t^{+}\right)=\pi / 2$.

Let $d(x, y)$ be the Euclidean distance between points $x, y \in \mathbb{R}^{n}$. We now work toward finding a lower bound on the distance between the points $r\left(t_{0}\right)$ and $r\left(t^{+}\right)$:

$$
\begin{aligned}
\left(r\left(t^{+}\right)-r\left(t_{0}\right)\right) \cdot T_{0} & =\int_{t_{0}}^{t^{+}}\left(r^{\prime}(t) \cdot T_{0}\right) d t \\
& =\int_{t_{0}}^{t^{+}}\left\|r^{\prime}(t)\right\| \cos (\theta(t)) d t .
\end{aligned}
$$


Let $K_{\Gamma}$ be the least upper bound of $K$ on $\Gamma$. Then, recalling that $d s / d t=\left\|r^{\prime}(t)\right\|$ gives

$$
\begin{aligned}
\left(r\left(t^{+}\right)-r\left(t_{0}\right)\right) \cdot T_{0} & =\int_{s\left(t_{0}\right)}^{s\left(t^{+}\right)} \cos (\theta(s)) d s \\
& =\int_{s\left(t_{0}\right)}^{s\left(t^{+}\right)} \frac{K_{\Gamma}}{K_{\Gamma}} \cos (\theta(s)) d s \\
& \geq \frac{1}{K_{\Gamma}} \int_{s\left(t_{0}\right)}^{s\left(t^{+}\right)} \frac{d \theta}{d s} \cos (\theta(s)) d s \\
& =\frac{1}{K_{\Gamma}} \int_{\theta\left(t_{0}\right)}^{\theta\left(t^{+}\right)} \cos (\theta) d \theta \\
& =\frac{1}{K_{\Gamma}} \int_{0}^{\pi / 2} \cos (\theta) d \theta \\
& =\frac{1}{K_{\Gamma}},
\end{aligned}
$$

where the inequality follows from Proposition 2.1. Similarly, $\left(r\left(t^{+}\right)-r\left(t_{0}\right)\right) \cdot T\left(t^{+}\right) \geq 1 / K_{\Gamma}$. Since $T_{0}$ and $T\left(t^{+}\right)$are orthogonal, it follows that $d\left(r\left(t^{+}\right), r\left(t_{0}\right)\right) \geq \sqrt{2} / K_{\Gamma}$. Similarly, $d\left(r\left(t^{-}\right), r\left(t_{0}\right)\right) \geq$ $\sqrt{2} / K_{\Gamma}$ and $d\left(r\left(t^{-}\right), r\left(t^{+}\right)\right) \geq 2 / K_{\Gamma}$. This leads to the following result.

Theorem 2.2. Let $\Gamma$ be a simple closed curve and let $K_{\star}$ be an upper bound for the curvature on $\Gamma$. Then, given a point $x_{0} \in \Gamma$, there exist points $x^{-}, x^{+} \in \Gamma$ such that $d\left(x_{0}, x^{-}\right), d\left(x_{0}, x^{+}\right) \geq \sqrt{2} / K_{\star}$ and $d\left(x^{-}, x^{+}\right) \geq 2 / K_{\star}$. Furthermore, the tangents to $\Gamma$ at $x^{-}$and $x^{+}$are orthogonal to the tangent at $x_{0}$.

By not requiring that the curve $\Gamma$ be closed, we obtain the following result, which will be useful in Section 4.

Theorem 2.3. Let $K_{\star}$ be an upper bound for the curvature on a curve $\Gamma$ which is given by $r: \mathbb{R} \rightarrow \mathbb{R}^{n}$, with $r^{\prime}$ nonvanishing. Suppose further that there exist $t^{-}, t_{0}, t^{+} \in \mathbb{R}$, with $t^{-}<t_{0}<t^{+}$such that the tangent at $x_{0}=r\left(t_{0}\right)$ is orthogonal to the tangents at $x^{-}=r\left(t^{-}\right)$and $x^{+}=r\left(t^{+}\right)$, and $r^{\prime}(t) \cdot r^{\prime}\left(t_{0}\right)>0$ for all $t \in\left(t^{-}, t^{+}\right)$. Then, $d\left(x_{0}, x^{-}\right), d\left(x_{0}, x^{+}\right) \geq \sqrt{2} / K_{\star}$ and $d\left(x^{-}, x^{+}\right) \geq 2 / K_{\star}$.

\section{Periodic solutions of differential equations}

Consider the differential equation

$$
x^{\prime}=f(x),
$$

where $f: \mathbb{R}^{n} \rightarrow \mathbb{R}^{n}$ is $C^{1}$. We denote by $x\left(t ; x_{0}\right)$ the solution to (3.1) which passes through $x_{0}$ at time $t=0$. Suppose $x\left(t ; x_{0}\right)$ is a nonconstant solution to (3.1). Noting that $x^{\prime \prime}=d\left(x^{\prime}\right) / d t=$ $\left(\partial\left(x^{\prime}\right) / \partial x\right)(d x / d t)=(\partial f / \partial x) f(x)$, it follows from (2.4) that the curvature at $x$ is

$$
\begin{aligned}
K(x) & =\frac{\|\| f(x)\left\|^{2} x^{\prime \prime}-\left(f(x) \cdot x^{\prime \prime}\right) f(x)\right\|}{\|f(x)\|^{4}} \\
& =\frac{\|\| f\left\|^{2}(\partial f / \partial x) f-\left(f^{T}(\partial f / \partial x) f\right) f\right\|}{\|f\|^{4}} .
\end{aligned}
$$


Thus, as long as the point $x$ is not an equilibrium of (3.1), the curvature at $x$ of the solution through $x$ can be precisely calculated without any explicit knowledge of the solution.

We now relate Theorem 2.2 to periodic solutions of ordinary differential equations.

Theorem 3.1. Let $\Gamma$ be the simple closed curve traced out by a nontrivial periodic solution of (3.1) and suppose that $\Gamma$ lies entirely in a region, where the curvature of solutions is bounded above by $K_{\star}$. Then, the conclusions of Theorem 2.2 hold.

Let $\Delta \subseteq \mathbb{R}^{n}$. Define

$$
K_{\Delta}=\sup _{x \in \Delta} K(x) .
$$

Then, any periodic solution to (3.1) which lies entirely in $\Delta$ has maximum curvature less than or equal to $K_{\Delta}$.

Corollary 3.2. If $\Delta$ contains no simple closed curve for which the maximum curvature is less than or equal to $K_{\Delta}$, then there are no nontrivial periodic orbits of (3.1) which lie entirely in $\Delta$.

Property 1 (The negative property). Let $K_{\star}$ be the maximum curvature of $f$ on a set which contains no equilibria. The set is said to have the negative property if it does not contain a point $x_{0}$ such that there are two corresponding points $x^{-}$and $x^{+}$satisfying $d\left(x_{0}, x^{-}\right), d\left(x_{0}, x^{+}\right) \geq$ $\sqrt{2} / K_{\star}$ and $d\left(x^{-}, x^{+}\right) \geq 2 / K_{\star}$, with $f\left(x^{-}\right)$and $f\left(x^{+}\right)$orthogonal to $f\left(x_{0}\right)$.

Corollary 3.3. If $\Delta$ has the negative property, then there are no periodic solutions to (3.1) contained entirely in $\Delta$.

\section{Omega limit points}

Theorem 4.1. Suppose $\Delta \subseteq \mathbb{R}^{n}$ has the negative property, and that $\varphi(t)$ is a forward trajectory of (3.1) which is contained entirely in $\Delta$. Then, the omega limit set of $\varphi$ consists entirely of equilibria which lie in the closure of $\Delta$, but not in $\Delta$.

Proof. Suppose $\bar{x}$ is an omega limit point of $\varphi(t)$, where $\varphi(t) \in \Delta$ for all $t \geq 0$, and that $f(\bar{x}) \neq 0$. Then, there is an increasing sequence of times $\left\{t_{n}\right\}$ which $\operatorname{limits}_{\text {to }} \infty$ such that $\lim _{n \rightarrow \infty} \varphi\left(t_{n}\right)=\bar{x}$. Then,

$$
\begin{aligned}
0 & =\lim _{n \rightarrow \infty}\left(\varphi\left(t_{n+1}\right)-\varphi\left(t_{n}\right)\right) \\
& =\lim _{n \rightarrow \infty} \int_{t_{n}}^{t_{n+1}} \varphi^{\prime}(t) d t \\
& =\lim _{n \rightarrow \infty} \int_{t_{n}}^{t_{n+1}} f(\varphi(t)) d t .
\end{aligned}
$$

Fix $\epsilon, \delta>0$ such that $f(y) \cdot f(z)>2 \epsilon$ if $y, z \in N_{\delta}$, the open ball centred at $\bar{x}$ with radius $\delta$. Choose $\tau>0$ so that $f(x(t ; y)) \cdot f(z)>\epsilon$ if $y, z \in N_{\delta}$ and $|t|<\tau$.

Taking the dot product of each side of (4.1) with a vector $v \in f\left(N_{\delta}\right)$ gives

$$
\lim _{n \rightarrow \infty} \int_{t_{n}}^{t_{n+1}}(f(\varphi(t)) \cdot v) d t=0
$$


For sufficiently large $n$, we have $\varphi\left(t_{n}\right) \in N_{\delta}$ and, therefore, $f(\varphi(t)) \cdot v>\epsilon$ for $\left|t-t_{n}\right|<\tau$. Thus,

$$
\int_{t_{n}}^{t_{n}+\tau}(f(\varphi(t)) \cdot v) d t>\epsilon \tau>0
$$

Hence, the integrand must oscillate between positive and negative for large enough $t$, in order that (4.2) be satisfied. Thus, for large enough $n$, there exist $t_{n}^{-}$and $t_{n}^{+}$with $t_{n}^{-}<t_{n}<t_{n}^{+}$, such that $f\left(\varphi\left(t_{n}^{-}\right)\right)$and $f\left(\varphi\left(t_{n}^{+}\right)\right)$are orthogonal to $v$. Furthermore, $t_{n}^{-}$and $t_{n}^{+}$can be chosen so that $f(\varphi(t)) \cdot v$ is positive on the interval with these endpoints.

The only restriction on $v$, thus far, is that $v \in f\left(N_{\delta}\right)$. In order for $n$ to be sufficiently large for $t_{n}^{-}$and $t_{n}^{+}$to be defined, it is only necessary that $\varphi\left(t_{n}\right) \in N_{\delta}$. (For large $n$, a change in the choice of $v$ will change the values of $t_{n}^{-}$and $t_{n}^{+}$but will not change the fact that they exist.) Now, fix $n$ to be sufficiently large that $\varphi\left(t_{n}\right) \in N_{\mathcal{\delta}}$, and choose $v=f\left(\varphi\left(t_{n}\right)\right)$.

Applying Theorem 2.3, with $\left(x_{0}, x^{-}, x^{+}\right)=\left(\varphi\left(t_{n}\right), \varphi\left(t_{n}^{-}\right), \varphi\left(t_{n}^{+}\right)\right)$, yields $d\left(x_{0}, x^{-}\right), d\left(x_{0}\right.$, $\left.x^{+}\right) \geq \sqrt{2} / K_{\Delta}$ and $d\left(x^{-}, x^{+}\right) \geq 2 / K_{\Delta}$. This contradicts the negative property, and so if $\bar{x}$ is an omega limit point, then we must have $f(\bar{x})=0$.

Since $\Delta$ satisfies the negative property, there are no equilibria in $\Delta$ and, therefore, $\bar{x}$ is not an element of $\Delta$. However, since $\bar{x}$ is an omega limit point of $\varphi$, it must be an element of the closure of $\Delta$, completing the proof.

Corollary 4.2. If $\Delta \subseteq \mathbb{R}^{n}$ is a compact set which has the negative property, then there are no forward trajectories contained entirely in $\Delta$.

Proof. Since $\Delta$ is compact, any forward trajectory contained entirely in $\Delta$ has a nonempty omega limit set $\Omega \subseteq \Delta$. By Theorem 4.1, $\Omega$ consists entirely of equilibria, but by the negative property $\Delta$ contains no equilibria, so $\Delta$ cannot contain an entire forward trajectory.

Corollary 4.3. Let $d$ be the diameter of a compact set $\Delta$ which contains no equilibria. If $d K_{\Delta}<2$, then any orbit starting in $\Delta$ leaves $\Delta$.

\section{Stability theorems}

Suppose $D \subseteq \mathbb{R}^{n}$ is positively invariant under (3.1). If $\Delta \subseteq D$ is compact and has the negative property, then by Corollary 4.2 there are no forward trajectories contained entirely in $\Delta$. If it is known that all solutions intersecting $D \backslash \Delta$ limit to an equilibrium, then we can conclude that all solutions in $D$ limit to an equilibrium giving the following result.

Theorem 5.1. Suppose $D$ is positively invariant, and $\Delta \subseteq D$ is a compact set which has the negative property. If all solutions in $D \backslash \Delta$ limit to an equilibrium, then all solutions in $D$ limit to an equilibrium.

Corollary 5.2. Suppose $D$ is positively invariant and contains a unique equilibrium $\bar{x}$. Suppose $\Delta \subseteq D$ is a compact set which has the negative property. If all solutions in $D \backslash \Delta$ limit $\bar{x}$, then $\bar{x}$ is globally stable in $D$.

We now consider the effect of making a change of variables. Suppose

$$
x=\alpha(y),
$$


where $\alpha: \mathbb{R}^{n} \rightarrow \mathbb{R}^{n}$ is a diffeomorphism. Then, $x^{\prime}=(\partial \alpha / \partial y) y^{\prime}$, and so

$$
\begin{aligned}
y^{\prime} & =\left[\frac{\partial \alpha}{\partial x}\right]^{-1} f(x) \\
& =\left[\frac{\partial \alpha}{\partial x}\right]^{-1} f(\alpha(y)) .
\end{aligned}
$$

If the set $\alpha^{-1}(\Delta)$ is compact and has the negative property for (5.2), then Corollary 4.2 implies solutions of (5.2) leave $\alpha^{-1}(\Delta)$, which then implies solutions of (3.1) leave $\Delta$. This allow Theorem 5.1 to be generalized as follows.

Theorem 5.3. Suppose $D$ is positively invariant under (3.1), $\Delta \subseteq D$ is compact and that all solutions in $D \backslash \Delta$ limit to an equilibrium. If there exists a diffeomorphism $\alpha: \mathbb{R}^{n} \rightarrow \mathbb{R}^{n}$ such that $\alpha^{-1}(\Delta)$ has the negative property for (5.2), then all solutions in D limit to an equilibrium.

\section{Discussion}

If it is known that the curvature of a vector field is bounded on a set, then it follows that the rate at which trajectories turn is bounded. A consequence of this is that a closed orbit has a minimum size. In particular, there exist points on the orbit which are separated by a distance at least twice as large as the reciprocal of the bound on the curvature.

This approach can be used to rule out periodic trajectories of a differential equation and to show that solutions which remain in a given set must limit to sets consisting of equilibria.

It may appear that this alone could be used to show global stability of an equilibrium in an invariant compact set, but there is a problem. Near an equilibrium the curvature is usually unbounded. This is true whenever the linearization at the equilibrium has at least two distinct eigenvalues.

Thus, in determining the global stability of an equilibrium, it would be necessary to deal with the behavior near the equilibrium in some other manner. For instance, one could use the linearization to obtain a quadratic form that could be used to show that a given neighborhood limits to the equilibrium. Then, by showing that the remainder of the compact set has the negative property, it would follow that the equilibrium is globally stable.

\section{References}

[1] J. Stewart, Multivariable Calculus: Early Transcendentals, Thomson Brooks/Cole, Belmont, Calif, USA, 2008. 


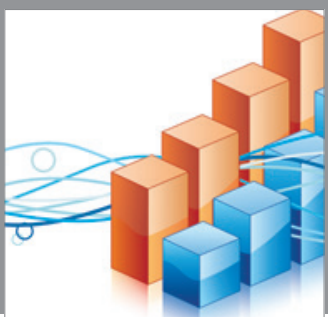

Advances in

Operations Research

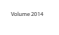

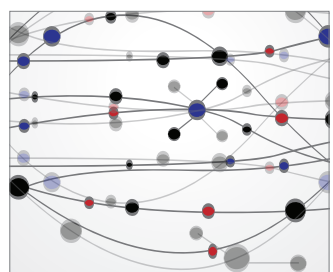

\section{The Scientific} World Journal
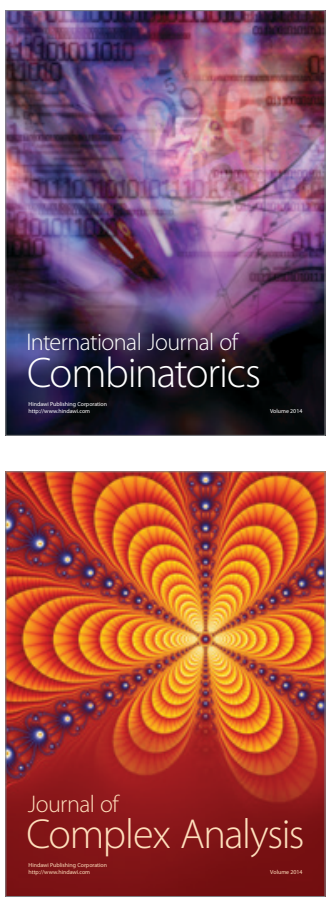

International Journal of

Mathematics and

Mathematical

Sciences
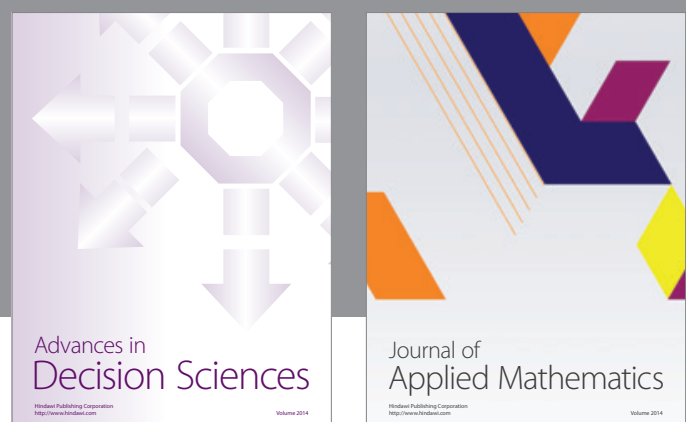

Journal of

Applied Mathematics
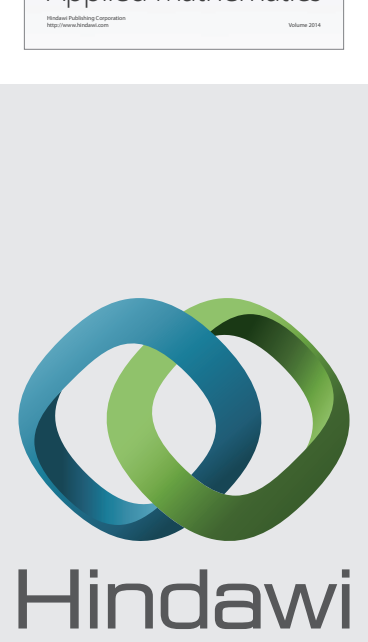

Submit your manuscripts at http://www.hindawi.com
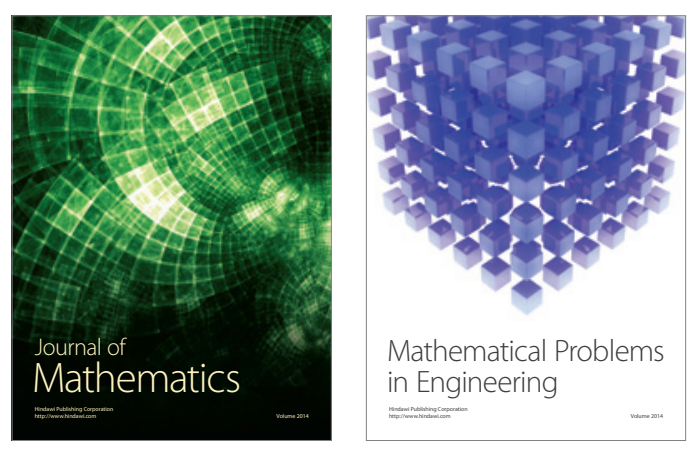

Mathematical Problems in Engineering
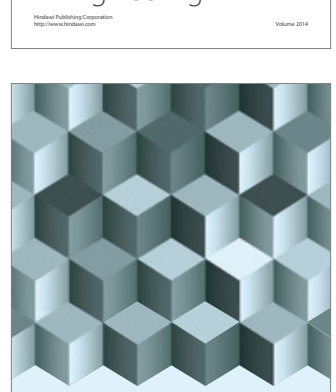

Journal of

Function Spaces
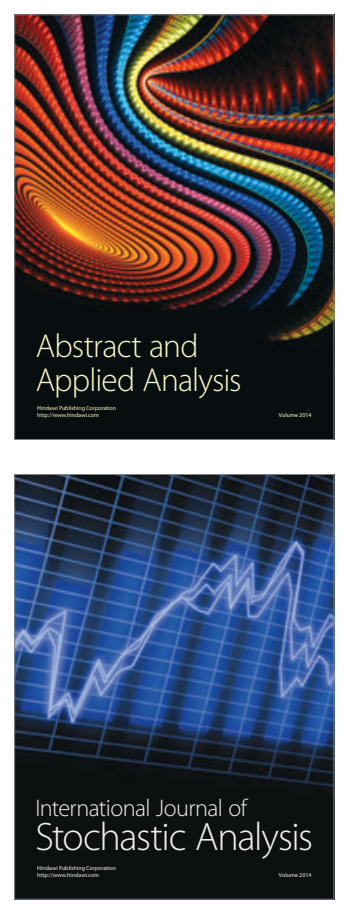

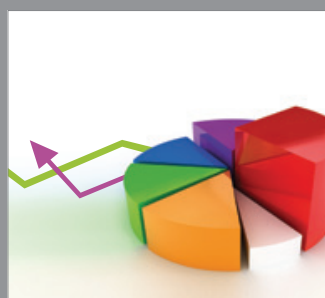

ournal of

Probability and Statistics

Promensencen
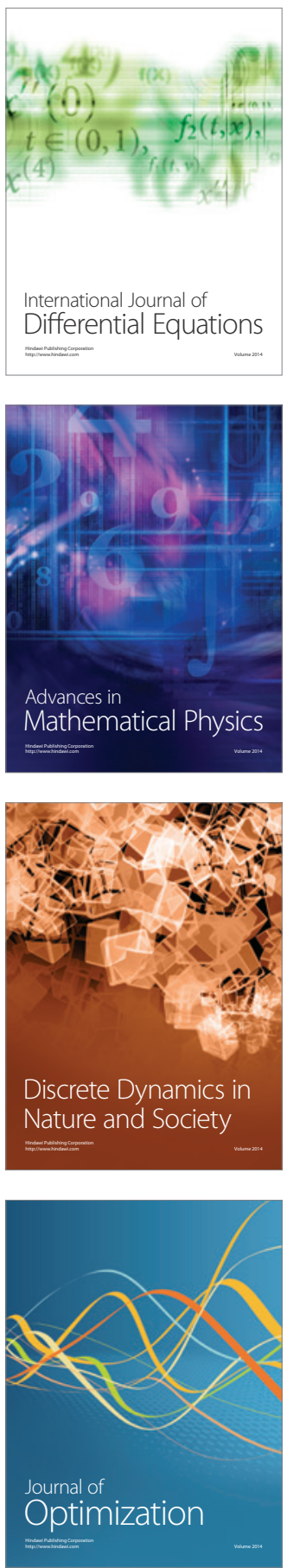